\title{
Condensation Model for Application of Computational Fluid Dynamics in Buildings
}

\author{
Cătălin Teodosiu, Viorel Ilie, and Raluca Teodosiu
}

\begin{abstract}
Moist air is an important factor influencing indoor air quality, human comfort, energy consumption of buildings and the durability of building materials. Consequently, the purpose of this work is to develop a numerical model of condensation phenomena for humid air in rooms. The model description is focusing on surface convection heat transfer and water vapor transport. The model is integrated in the computational fluid dynamics (CFD) technique. As a result, the whole numerical CFD model can be used to predict airborne moisture in rooms, taken also into consideration condensation of humid air on cold surfaces. The performance of the numerical model is analyzed using experimental data for water vapor condensation on a glazed wall in a ventilated test room. The experimental-numerical comparisons show the capability of the model to predict in a realistic manner the condensate appearance and its distribution on cold surfaces. Accordingly, the results of this study can be applied in several fields: buildings (indoor air quality, thermal comfort and energy consumption), industry (microclimate control and technological conditions), and vehicles (freezing prediction on the windshield),
\end{abstract}

Index Terms-CFD simulations, full scale test cell, humidity modeling, surface condensation model.

\section{INTRODUCTION}

It is known that most of building construction problems are related to water (liquid phase or vapor phase). For instance, humid indoor air can lead to condensation on windows or even moisture stains on walls, ceilings, furniture, etc. Too humidity in indoor air can also promote the growth of mould. On the other hand, when indoor humidity is too low, static electricity and electronic devices malfunction are among common problems. In addition, there are some applications (museums, hospitals, pools, specific industrial fields, e.g. electronics, precision mechanics, pneumatic control systems, complex printing processes, food, pharmaceutical, etc.) where the indoor air humidity levels and condensation issues are the main concerns in order to correctly manage the indoor environment conditions for occupants or for technological purposes.

It is worthwhile to mention that condensation or mould growth occur locally. These phenomena deeply depend on

Manuscript received August 4, 2014; revised October 24, 2014. This work was supported by the Romanian National Authority for Scientific Research, CNDI-UEFISCDI under Grant PN-II-ID-JRP-RO-FR-2012-0071.

C. Teodosiu and R. Teodosiu are with the Thermo-Hydraulic and Protection of the Atmosphere Systems Department, Technical University of Civil Engineering, Bucharest 020396, Romania (e-mail: cteodosiu@yahoo.com, ralucahohota@yahoo.com).

V. Ilie is with the Doctoral Studies Department, Technical University of Civil Engineering, Bucharest 020396, Romania (e-mail: viorel_ilie_88@yahoo.com). local psyhrometric conditions (temperature, humidity) but also on local air flows. Consequently, detailed moisture-heat-airflow models are required. In this context, the CFD (Computational Fluid Dynamics) approach can be the appropriate solution as this method allows detailed predictions of temperature and velocity fields in rooms. Indeed, the CFD technique became a routinely tool in civil engineering for predicting air movement in enclosures [1]. Moreover, the CFD models are increasingly employed nowadays to evaluate detailed heat, air and moisture transfer mechanisms in indoor environments [2]-[5]. Unfortunately, the CFD studies dealing with humidity aspects including condensation in buildings are generally lacking.

As a result the purpose of this study is to propose a model that takes into account wall surface condensation phenomena in CFD simulations for buildings.

\section{WATER VAPOR CONDENSATION MODELING}

The model for the surface condensation of the water vapor is based on the following simplifying hypothesis: the humid air is an ideal gas, being a perfect mixture of 2 perfect gases (dry air and water vapor); there is no chemical reaction between these two gases; the binary mixture is an incompressible Newtonian fluid, the heat and mass transfer interactions (Soret and Dufour effects, respectively) are negligible; the surface on which condensation takes place is impermeable in terms of absorption-desorption phenomena; the quantity of condensed vapor is low (as it usually happens in buildings); the amount of condensate is removed from the computational domain.

The key factor for the surface condensation modeling is the surface temperature. The accurate calculation of this parameter (both as value and distribution) is crucial for the appropriate prediction of surface condensation and its development. For this reason, the correct approach to evaluate the surface temperature should take into account the sensible heat transfer (conduction, convection and radiation) and latent heat transfer of that surface.

Concerning the surface convection heat transfer and water vapor transport, the model is based on the methodology suggested by International Energy Agency [6]. Accordingly, the water vapor transport in air is mainly carried out by convective movements, the exception being around the surfaces where the diffusion plays the main role. As a result, the last layer near the walls is crossing through the diffusion mechanisms. This is expressed in the equation of the condensed vapour density flux as follows:

$$
\Phi_{\text {vap.cond }}=\beta\left(P_{\text {vap.air }}-P_{\text {vap.surface }}\right)
$$

where $\beta$ ( $\mathrm{s} / \mathrm{m})$ - proportionality coefficient that describes the 
water vapor diffusion between the indoor air and the walls surface, $p_{\text {vap.air }}$ is the "vapor pressure within the indoor air" and $p_{\text {vap.surface }}$ is the "vapor pressure on the wall surface".

The correlation between the convective transfer and the coefficient $\beta$ is [6]:

$$
\beta=\frac{h_{c}}{c_{p} R T \rho}\left[\frac{c_{p} R T \delta_{p}}{\lambda}\right]^{0.67}
$$

where $h_{c}$ is the convective heat transfer coefficient, $c_{p}$ is the specific heat capacity of water vapor and $\delta_{p}$ is the water vapor permeability (coupled to the water vapor pressure gradient).

Equation (2) can be rewritten, for applications in the field of thermal building, as a relationship that depends mainly on the convective heat transfer coefficient [6]:

$$
\beta=\frac{h_{c} \delta_{p}}{\lambda}=7,4 \times 10^{-9} h_{c}
$$

The convective heat transfer coefficient is calculated in our model taking into account the triangular faces (on the walls) of the discretization mesh and the centers of the first tetrahedral discretization elements within the indoor air (viscous zone), supposing that an unstructured mesh is used for the discretization of the computational domain in the CFD model. Therefore, for the viscous sublayer (e.g. cell with a triangle of area $S_{F}$ and temperature $T_{F}$ ), the convective heat exchange (Newton's law) can be written in the following manner:

$$
\Phi=h_{c}\left(T_{w}-T_{a i r}\right) S=h_{c}\left(T_{F}-T_{C 0}\right) S_{F}
$$

where $T_{w}$ - wall surface temperature, $T_{a i r}$ - air temperature and $S$ - surface; $T_{C 0}$ - first barycenter tetrahedron (near the wall) temperature, based on the notations of Fig. 1: there are triangles on the border with centroids $\mathrm{F}_{\mathrm{i}}$; in addition, each triangle (i) represents the geometric basis of a tetrahedron with the barycenter $\mathrm{CO}_{i}$; in the end, the mesh of the interior volume is characterised by a centroid $\mathrm{C}_{\mathrm{i}}$, node in which the calculation is performed.

As a result, we make use of the compute nodes $\mathrm{F}_{\mathrm{i}}$ for the surface treatment, taking also into account the centers $\mathrm{CO}_{\mathrm{i}}$ in order to make the connection between surfaces and the internal computing field.

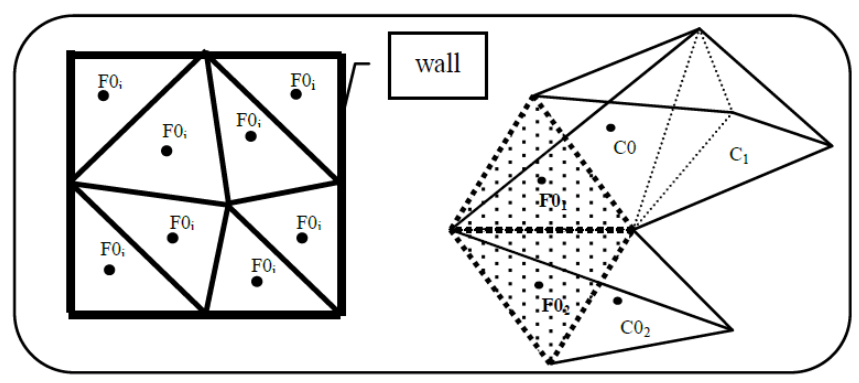

Fig. 1. Surface and boundary layer discretization (unstructured mesh):

$\mathrm{FO}_{\mathrm{i}}$ - border node; $\mathrm{CO}_{\mathrm{i}}-$ first tetrahedron node; $\mathrm{C}_{\mathrm{i}}-$ internal volume node.

Moreover, the viscous region near the solid borders (walls) is characterised in terms of heat exchanges by heat diffusion (conduction), the convection having a much less important role. For this reason, the heat flux density can be taken into account by the classical Fourier law:

$$
\varphi=-\lambda_{\text {air }} \overrightarrow{\operatorname{grad}} \vec{T} \vec{n}=\lambda_{\text {air }}\left(\frac{\partial T}{\partial n}\right)=h_{c}\left(T_{F}-T_{C 0}\right) \Rightarrow h_{c}=\frac{\lambda_{\text {air }}}{\left(T_{F}-T_{C 0}\right)}\left(\frac{\partial T}{\partial n}\right)
$$

where $\lambda_{\text {air }}$ is the thermal conductivity of the humid air and temperature gradient is computed as follows:

$$
\left(\frac{\partial T}{\partial n}\right)=\frac{\left(T_{F}-T_{C 0}\right)}{S_{F}} \alpha
$$

where $S_{F}$ represents the area of a triangle and $\alpha$.

$$
\alpha=\frac{\overrightarrow{S_{F}} * \overrightarrow{S_{F}}}{\overrightarrow{S_{F}} * \overrightarrow{s_{0}}} ; \text { with } s_{0}=\left(\operatorname{coord}_{F}-\operatorname{coord}_{C 0}\right)
$$

Consequently, the coefficient $\beta$ in (3) always varies with the convective heat transfer coefficient (derived in turn from heat exchanges calculation between the fluid and the walls in the CFD simulations). As an example, for a cell that has one face on the wall, we realize the computation of the convective heat transfer coefficient as a function of: temperature difference between the center of the triangle (on the wall) and the centroid of the tetrahedron which contains the triangle; distance between the center of the triangle and the centroid of the tetrahedron enclosing the triangle; the surface of the triangle; thermal conductivity of the moist air located in the tetrahedron centroid.

As soon as the coefficient $\beta$ is solved, the mass flow rate of water vapor condensed on the surface $m_{\text {vap.cond }}$ is calculated, for each triangle that is positioned on the solid boundary of the computational domain, in the following manner:

$$
\dot{m}_{\text {vap.cond }}=\frac{d m_{\text {liq.sufface }}}{d t}
$$

If

$$
\begin{aligned}
& P_{\text {vap }}-P_{\text {vap.sat }}>0 \\
& \quad \dot{m}_{\text {liq.surface }}=7,4 \times 10^{-9} h_{c} S_{F_{i}}\left(P_{\text {vap. }}-P_{\text {vap.sat }}\right)
\end{aligned}
$$

Else

$$
\dot{m}_{\text {liq.surface }}=0
$$

where $m_{\text {liq.surface }}$ is the condensed vapour flux, calculated using (1), $p_{\text {vap.sat }}$ is the saturation pressure of water vapor and $p_{\text {vap. }}$ is the partial pressure of water vapor. These pressures were calculated using correlations proposed in the literature [7].

In this way, based on the mass flow rate of water vapor condensed on the surface, the connection with the volume computation is achieved, by means of two balances: mass balance, where the condensate flow rate is removed from the computational domain through the intermediary of the source term that appears in the water vapor conservation equation of the CFD model (see Section III), and energy balance, where sink terms that correspond to latent heat of vaporisation and sensible heat of the condensed water vapour amount (removed from the computational domain) are added in the CFD model:

$$
H_{\text {cond. }}^{\cdot}=\dot{m}_{\text {vap.cond }}\left[L_{v a p}+c_{p} *\left(T_{F i}-T_{\text {ref }}\right)\right]
$$

where $H_{\text {cond }}$ represents the total enthalpy of condensed water 
vapor, taking into account $L_{v a p}$ - water latent heat of vaporization (with $T_{\text {ref }}$ - reference temperature).

Finally, to better understand the implementation of mass and energy source/sink terms in the equations of the CFD model, we show in Fig. 2 a simplified scheme.

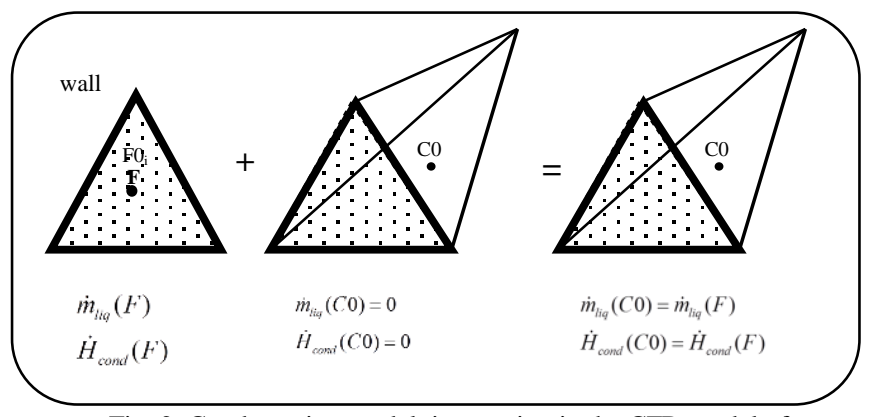

Fig. 2. Condensation model: integration in the CFD model of mass and energy source/sink terms.

It should be said that the model of surface condensation presented above can be easy extrapolated for predictions of volume condensation, using the same approach based on mass and energy source/sink terms added in the CFD simulations (equation of water vapor conservation and equation of energy, respectively).

Concerning the heat conduction in walls of the CFD computational domain, the condensation model in the present form takes into consideration one-dimensional simulations (heat conduction only in the wall-normal direction). The walls are considered as "monolayer" components from the point of view of conductive heat transfer, using the thermal resistance to compute the heat transfer across them. Further work should include two-dimensional calculations of the conduction heat flux through walls to better assess the influence of thermal bridges on surface temperature of walls.

On the other hand, there are several methods to calculate the radiation heat transfer in conjunction with CFD technique: discrete transfer radiation model (DTRM), P-1 radiation model (the simplest case of the more general $\mathrm{P}-\mathrm{N}$ radiation model), discrete ordinates (DO) radiation model, Rosseland radiation model and surface-to-surface $(\mathrm{S} 2 \mathrm{~S})$ radiation model The formulation of these models can be found in fundamental books on thermal radiation and its modeling [8]. Any of these methods can be used to account for the radiative heat transfer within the condensation model proposed here for CFD simulations.

\section{INTEGRATION OF CONDENSATION MODEL IN CFD}

In order to integrate in CFD simulations the condensation model developed here, an equation describing the conservation of the water vapor mass fraction should be compulsory added to the basic equations governing a turbulent non-isothermal airflow. This equation is written in a tensor notation as:

$$
\rho \frac{\partial}{\partial x_{i}}\left(u_{i} m_{i^{\prime}}\right)+\frac{\partial}{\partial x_{i}} J_{i^{\prime}, i}=S_{i^{\prime}}
$$

where the left-hand side terms stand for the convective term ( $\rho$ - density of the humid air, $x_{i}$ - spatial coordinate, $u_{i}$ - velocity component in $i$ direction, $m_{i}$, - water vapour mass fraction) and diffusion term respectively $\left(J_{i, i},-\right.$ water vapour diffusion flux), while the right-hand side term $S_{i}$, represents source/sink term (e.g. the term computed based on the condensation model as explained above).

The diffusive term in (9) takes into account both classical aspects of diffusion, molecular and turbulent:

$$
\frac{\partial}{\partial x_{i}} J_{i^{\prime}, i}=\rho \frac{\partial}{\partial x_{i}}\left(D_{i^{\prime}, m} \frac{\partial m_{i^{\prime}}}{\partial x_{i}}\right)-\frac{\partial}{\partial x_{i}}\left(-\overline{u_{i}^{\prime} m_{i^{\prime}}^{\prime}}\right)
$$

where $D_{i, m}$ stands for the water vapor molecular diffusion coefficient and $u_{i}{ }^{\prime} m_{i}^{\prime}$, signifies the turbulent mass flux of water vapor, $u_{i}$ ' being the velocity fluctuation.

Regarding the molecular diffusion, this can be classically fulfilled by taking into account the Fick's first law (diffusion due to concentration gradients) by means of a moisture diffusion coefficient in the air. It can be used a constant value for the diffusion coefficient of water vapor in air since its variations as a function of temperature and viscosity are insignificant in the field of thermal building.

On the other hand, the turbulent mass flux of water vapor is estimated based on the turbulence model used in CFD simulations.

It can be noticed that the convection-diffusion equation for the conservation of the water vapour (9) is similar to classic transport CFD equations. In fact, it has the same form as the other equations describing in the CFD model the conservation of a variable within the computational field (e.g. mass, momentum, energy, turbulent quantities). For this reason, (9) can be numerically solved by the same methods used for all convection-diffusion equations of the CFD model.

\section{Condensation Model Application: CASE Study}

The condensation model and its integration in CFD simulations are exemplified for water vapor condensation on a glazed wall in a ventilated test room. In fact, the numerical results are compared to experimental data completed in a full scale test cell $\left(3.1 \times 3.1 \times 2.5 \mathrm{~m}^{3}\right)$ - test cell 1 in Fig. 3 .

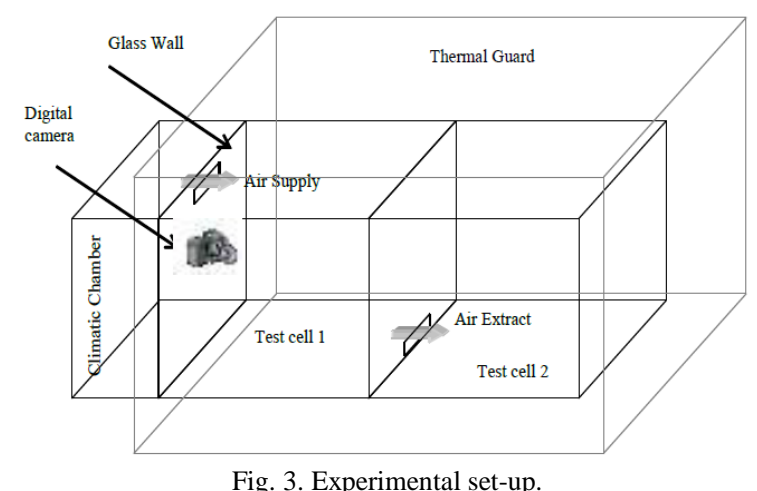

The experimental set-up contains the following main elements: 2 identical rooms (test cell 1 and test cell 2 - Fig. 3); 5 thermal guard spaces (maintained at a constant temperature of $21 \ldots 22^{\circ} \mathrm{C}$ ); 1 climatic chamber where the temperature can vary between $-10^{\circ} \mathrm{C} \ldots+40^{\circ} \mathrm{C}$ (a single glazed "façade" separates this space from the test cell 1); mixing ventilation 
system for test cell 1; supply air humidification system (based on 2 steam immersed electrode humidifiers); condensation qualification system (based on digital camera with high quality images; the camera is located in the climatic chamber) The exhaustive experimental description can be found in [9].

The experimental test configurations taken into account are presented below. The air inlet characteristics are given in Table I. Concerning the thermal boundary conditions, the air temperature in the climatic chamber adjacent to the glass wall was maintained at $11.7^{\circ} \mathrm{C}$ during the investigations.

TABLE I: EXPERIMENTAL CONDITIONS (AIR INLET CHARACTERISTICS)

\begin{tabular}{llll}
\hline $\begin{array}{l}\text { Archimedes } \\
\text { number }\end{array}$ & $\begin{array}{l}\text { Reynolds } \\
\text { number }\end{array}$ & $\begin{array}{l}\text { Inlet moisture } \\
\text { content }[\mathrm{g} / \mathrm{kg}]\end{array}$ & $\begin{array}{l}\text { Inlet air } \\
\text { temperature }\left[{ }^{\circ} \mathrm{C}\right]\end{array}$ \\
\hline 0.0032 & 19187 & $10.2-13.1$ & 41.3 \\
\hline \hline
\end{tabular}

The principal characteristics of the CFD model used for the implementation of the condensation methodology developed here are presented in Table II. This model has been assembled on the basis of a general-purpose, finite-volume, Navier-Stokes solver (Fluent version 15.0.0). It is worthwhile to mention that the condensation modeling has been integrated in the CFD simulations using the code facility, called "user defined functions" (UDF). These "UDfs" have been programmed following the model description (see Section II).

TABLE II: MAIN FEATURES OF THE CFD MODEL

\begin{tabular}{|c|c|}
\hline Flow & $\begin{array}{l}\text { Three-dimensional, steady, non-isothermal, } \\
\text { turbulent }\end{array}$ \\
\hline $\begin{array}{l}\text { Computational } \\
\text { domain } \\
\text { discretization }\end{array}$ & $\begin{array}{l}\text { Finite volumes, unstructured mesh (tetrahedral } \\
\text { elements) }\end{array}$ \\
\hline $\begin{array}{l}\text { Turbulence } \\
\text { model }\end{array}$ & Two-equation model ("realizable" k- $\varepsilon$ model) \\
\hline $\begin{array}{l}\text { Near-wall } \\
\text { treatment }\end{array}$ & Two-layer model \\
\hline Radiation model & discrete ordinates (DO) radiation model \\
\hline $\begin{array}{l}\text { Numerical } \\
\text { resolution }\end{array}$ & $\begin{array}{l}\text { Segregated implicit solver } \\
\text { Diffusion terms: second-order central-difference } \\
\text { scheme } \\
\text { Convective terms: second-order upwind scheme } \\
\text { Velocity_pressure coupling: SIMPLE algorithm } \\
\text { Convergence acceleration: algebraic multigrid }\end{array}$ \\
\hline
\end{tabular}

Finally, it is worthwhile to mention that the radiative heat transfer modeling considered the humid air in the room as a non-participating radiation medium. This hypothesis has led to satisfactory results [10] in spite of the fact that most air in the condensation model contains water vapor, known as absorbing constituent in the infrared. This assumption remains valid even at the saturated state of the humid air [11], a situation that usually leads to the appearance of surface condensation. In addition, all the surfaces of the computational domain are considered to be gray-diffuse surfaces in the condensation model in order to evaluate the radiative heat fluxes with their multiple reflections that take place in rooms. This is a usual hypothesis in the field of thermal building for the calculation of radiative exchanges.

\section{RESULTS}

The numerical results put in evidence the condensate distribution on the inner surface of the glazed wall (mass of liquid). The data are presented for the following values of supply air moisture content: $10.2 \mathrm{~g} / \mathrm{kg}$ (case I) and $13.1 \mathrm{~g} / \mathrm{kg}$ (case II).

Before submitting the qualitative experimental-numerical comparison in terms of condensation on the cold wall of the cell test, we first analyze the specific humidity of extract air (Table III).

TABLE III: SPECIFIC HUMIDITY OF EXTRACT AIR (CASE I)

\begin{tabular}{ccc}
\hline \hline $\begin{array}{c}\text { Experimental } \\
(\mathrm{g} / \mathrm{kg})\end{array}$ & $\begin{array}{c}\text { Numerical } \\
(\mathrm{g} / \mathrm{kg})\end{array}$ & $\begin{array}{c}\text { Relative error } \\
\text { experimental-numerical }(\%)\end{array}$ \\
\hline 10.66 & 10.83 & 1.6 \\
\hline \hline
\end{tabular}

There are small deviations between measures and numerical results $(1.6 \%)$. This proves that the room balance in terms of water vapor quantity in the humid air is correctly achieved. This also indicates that the mass of liquid that appears by condensation on the glazed wall is correctly predicted in the CFD model.

Concerning the appearance of the condensate and its distribution on the glass wall, Fig. 4 shows condensate traces in the areas A3 and F3 in the two pictures (numerical and experimental), for case I. In addition, there is an uniform distribution of condensation on the glass surface, which is explained by the symmetric jet, the side walls maintained at the same temperature, the constancy of temperature in the climatic chamber and border effects.

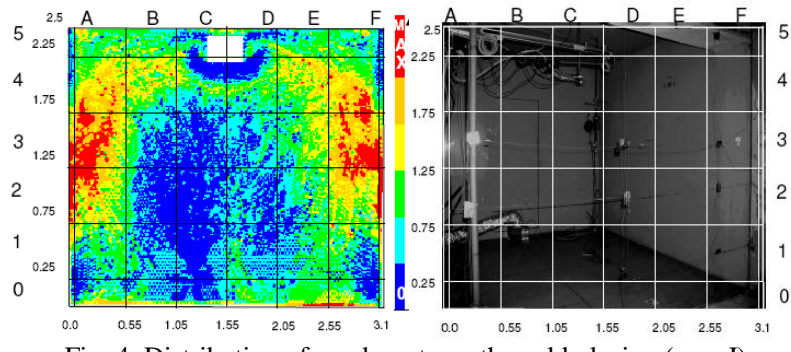

Fig. 4. Distribution of condensate on the cold glazing (case I).

For the other condensation test (case II), the water vapor amount is more important than the first situation taken into consideration (case I). Consequently, this allows acquiring more eloquent images of condensate distribution on the window (Fig. 5).
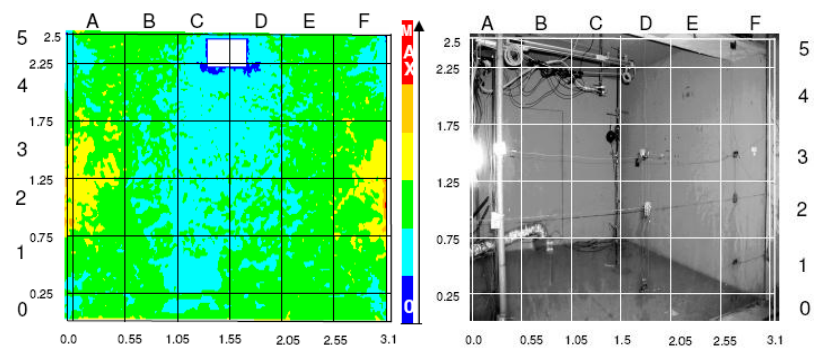

Fig. 5. Distribution of condensate on the cold glazing (case II).

It can be noticed good numerical predictions of condensation distribution: the surfaces covered by condensate are located in the numerical model in a more than satisfactory manner compared to experimental condensate distribution.

\section{CONCLUSION}

The numerical model presented in this study is capable of 
estimating the appearance of the condensate and its distribution on cold surfaces.

This conclusion is based on experimental validation using water vapor condensation tests on a glazed wall in a ventilated test cell.

On the other hand, it should be remembered that, at the base, the condensation phenomenon are quasi instantaneous so unsteady treatment is necessary if we want to achieve an eloquent description of the mechanism. As a result, transient simulations should be carried out in order to be able to predict the exact moment of condensate appearance and its evolution in time. It should be mentioned that unsteady simulations already completed, based on the condensation model presented here, have led to relevant results. Nevertheless, this study proves that computations in steady state conditions for the phenomenon of humid air condensation on cold surfaces can lead to realistic results. This is an important benefit, taken into account the important decrease of central processing unit (CPU) time in comparison to unsteady simulations.

In addition, further work should be performed in order to attach an evaporation model to this numerical approach. On the other hand, the experimental technique must be improved in order to determine the exact quantity of condensate on the glazed wall and its development.

\section{REFERENCES}

[1] D. N. Sørensen and P. V. Nielsen, "Quality control of computational fluid dynamics in indoor environments," Indoor Air, vol. 13, no.1, pp 2-17, March 2003.

[2] C. Teodosiu, R. Hohota, G. Rusaouën, and M. Woloszyn, "Numerical prediction of indoor air humidity and its effect on indoor environment," Building and Environment, vol. 38, no. 5, pp. 655-664, May 2003.

[3] L. H. Mortensen, M. Woloszyn, C. Rode, and R. Peuhkuri, "Investigation of microclimate by CFD modeling of moisture interactions between air and constructions," Journal of Building Physics, vol. 30, no. 4, pp. 279-315, 2007.

[4] M. Van Belleghem, H. J. Steeman, M. Steeman, A. Janssens, and M. de Paepe, "Sensitivity analysis of CFD coupled non-isothermal heat and moisture," Building and Environment, vol. 45, no. 11, pp. 2485-2496, January 2010.

[5] H. Huang, S. Kato, R. Hu, and Y. Ishida, "Development of new indices to assess the contribution of moisture sources to indoor humidity and application to optimization design: Proposal of $\mathrm{CRI}_{(\mathrm{H})}$ and a transient simulation for the prediction of indoor humidity," Building and Environment, vol. 46, no. 9, pp. 1817-1826, September 2011.

[6] Condensation and Energy. Energy Conservation in Buildings and Community Systems Programme, International Energy Agency Annex 14, Report Annex 14, vol. 1-1991.

[7] K. Raznjevic, Thermodynamic Tables and Diagrams (in French), Paris, France: Eyrolles, 1970.

[8] M. F. Modest, Radiative Heat Transfer, London, UK: Academic Press, 2003

[9] R. Hohota, "Moisture modeling in a CFD code (low velocity in large cavity) - comparison with the experimental (in French)," Ph.D. Thesis, INSA Lyon, France, 2003.

[10] M. Tabarki and S. B. Mabrouk, "The coupling in transient regime between the modelings of thermal and mass transfers inside a heated room and its radiator," Heat Mass Transfer, vol. 48, pp. 1889-1901, 2012

[11] F. Allard, "Contribution to the study of heat transfer in thermally driven cavities at high Rayleigh number (in French)," Ph.D. Thesis, INSA Lyon, France, 1987.

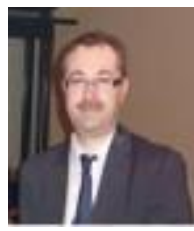

Cătălin Teodosiu was born in Bucharest, Romania, on July 22, 1972, who received the B.E. degree in civil engineering in 1996, the M.Sc. degree in energy management in 1997 from the Technical University of civil engineering (Building Services Faculty), Bucharest, Romania and the Ph.D. degree in civil engineering in 2001 from the National Institute of Applied Science (INSA) of Lyon, France. Between 1996 and 1998, he was an assistan professor and from 2004 till now he has been a lecturer and an associate professor. He joined the Thermo-Hydraulic and Protection of the Atmosphere Systems Department, Faculty of Building Services and Equipment, Technical University of Civil Engineering, Bucharest. He performed teaching and research activity also in France, as an assistant lecturer and a researcher, from 1998 to 2004 in the University Claude Bernard - Lyon I, INSA of Lyon and CETHIL - The Center for Thermal Sciences of Lyon. He is the author and co-author of four books and university courses. He published over fifty-five articles in international and national journals and papers in peer-reviewed international conferences proceedings. $\mathrm{He}$ was a participant at 8 international research-development-innovation projects (four of which as project manager); one national research project financed by European Union; twenty-one national research-development-innovation projects (two as project responsible). He is also co-author at two national technical regulatory documents. $\mathrm{He}$ is an energy building auditor (Ministry of Regional Development and Tourism - MDRT authorisation) and technical expert for HVAC installations (MDRT authorisation). His current research interests include CFD (Computational Fluid Dynamics) modeling, focusing on turbulence models and integrated heat-airflow-moisture models; building simulation; high efficiency buildings.

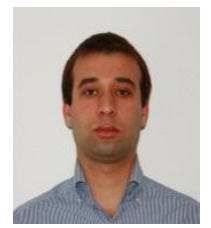

Viorel Ilie was born in Braila, Romania, on January 6 , 1988, who received the B.E. degree in civil engineering in 2011 and the M.Sc. degree in energy efficient equipment for buildings in 2013 from the Technical University of Civil Engineering (Building Services Faculty), Bucharest, Romania. He is a Ph.D. student at the Thermo-Hydraulic and Protection of the Atmosphere Systems Department, Faculty of Building Services and Equipment, Technical University of Civil Engineering, Bucharest since 2013.

His main research field covers computational fluid dynamics (CFD) modeling and ventilation systems efficiency.

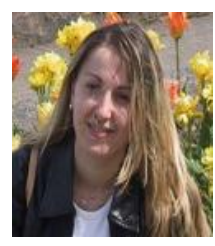

Raluca Teodosiu was born in Bucharest, Romania, on April 11, 1976, who received the B.E. degree in civil engineering in 1999 , the M.Sc. degree in civil engineering in 2000 from the Technical University of Civil Engineering (Building Services Faculty), Bucharest, Romania and the Ph.D. degree in civil engineering in 2004 from the National Institute of Applied Science (INSA) of Lyon, France. She was an engineer at Institute for Studies and Power Engineering (ISPE), Bucharest, Romania (1999-2000). She was also a research engineer at CETHIL - The Center for Thermal Sciences of Lyon (2000-2003) and an assistant lecturer and researcher at University Claude Bernard - Lyon I and CETHIL, France from 2003 to 2004. She joined in 2004 the Thermo-Hydraulic and Protection of the Atmosphere Systems Department (Faculty of Building Services Engineering - Technical University of Civil Engineering Bucharest). She published over 40 articles in international and national journals and papers in peer-reviewed international conferences proceedings. She is the co-author at 1 national technical regulatory documents. She was a member of one international research-development-innovation projects; one national research project financed by European Union; seven national research-development-innovation projects. She is an energy building auditor (Ministry of Regional Development and Tourism - MDRT authorisation) Her current research interests include CFD (Computational Fluid Dynamics) modeling, experimental investigations concerning indoor air quality; thermal-aeraulic behavior of buildings; modeling energy consumption of buildings.

R. Teodosiu is a scientific secretary of Energy Auditors Order Romania, a member of Romanian Association of Building Services Engineers Bucharest Subsidiary and Scientific and Advisory Committee RCEPB (Romanian Conference on Energy Performance of Buildings) in 2011, 2012, 2013 and 2014 